\title{
Hernia de Garengeot, diagnóstico ecográfico y manejo quirúrgico de una entidad infrecuente
}

\section{De Garengeot's Hernia, Ultrasound Examination and Surgical Management of an Infrequent Entity}

\author{
Felipe González ${ }^{1}$ Cristián Cavalla ${ }^{2}$ Guillermo Aguilera ${ }^{3}$ Gerhard Franz ${ }^{3}$
}

\author{
${ }^{1}$ Radiólogo, Clínica Alemana de Santiago, Vitacura, Chile \\ ${ }^{2}$ Cirujano digestivo, Clínica Alemana de Santiago, Vitacura, Chile \\ ${ }^{3}$ Residente de Radiología, Clínica Alemana de Santiago- Universidad \\ Del Desarrollo, Vitacura, Chile
}

Rev Argent Radiol 2018;82:86-87.

Estimados editores,

La hernia de Garengeot es una entidad rara, descrita por primera vez en 1731 por el cirujano francés Rene Jacques Croissant de Garengeot, ${ }^{1}$ quien reporta durante una exploración quirúrgica la presencia del apéndice cecal contenido dentro de una hernia femoral. Esas hernias ingresan al canal femoral ubicándose en el triángulo de Scarpa, destacándose por un mayor riesgo de complicación debido al pequeño tamaño de su anillo. Generalmente contienen intestino delgado $\mathrm{y} / \mathrm{o}$ tejido adiposo visceral, siendo infrecuente la presencia del apéndice cecal $(0,5-1 \%$ de los casos) y más infrecuente aún la concomitancia con apendicitis aguda $\left(0,08-0,13 \%\right.$ de los casos). ${ }^{2,3}$ Se presenta el caso de una mujer de 75 años con antecedentes de hipertensión arterial, dislipidemia, sarcoidosis, y derivación biliodigestiva reciente por estenosis de la vía biliar, en control y tratamiento. Consultó en el servicio de urgencia por cuadro de un día de aumento de volumen en región inguino-crural derecha asociado a dolor EVA 2/10, sin otro síntoma a la anamnesis dirigida. Cuando se realizó el examen físico, la paciente estaba afebril, destacando una masa sensible no reductible de la región crural derecha, sin otros hallazgos positivos que consignar. Los exámenes de laboratorio demostraron hematocrito $39,1 \%, \mathrm{Hb}$, $12,9 \mathrm{~g} / \mathrm{dL}$, leucocitos $9.600 \mathrm{u} / \mathrm{mm} 3$, neutrófilos $80 \%$, plaquetas $242.000 \mathrm{u} / \mathrm{mm} 3$, VHS $14 \mathrm{~mm} / \mathrm{h}$. Por la sospecha clínica de una hernia crural atascada se realizó una ecografía abdominal e inguino-crural, en la cual se observó un saco herniario en relación a los vasos femorales derechos (-Fig. 1), no reductible al paso del transductor, que presentaba contenido líquido y adiposo, ése último con aumento de su ecogenicidad debido a cambios inflamatorios. En su interior destacaba además la presencia de una estructura tubular de

received

April 27, 2017

accepted

September 9, 2017

published online

April 27, 2018
Address for correspondence Dr. Guillermo Esteban Aguilera Silva, Santiago, Clínica Alemana de Santiago, Vitacura, Universidad del Desarrollo, Chile (e-mail: gaguileras@alemana.cl).

aproximadamente $10 \mathrm{~mm}$ de diámetro, no compresible, que no presentaba vascularización al modo Doppler color, y que se interpretó como el apéndice cecal con cambios inflamatorios (-Fig. 2). Con ese diagnóstico, la paciente ingresó al pabellón quirúrgico sin realizarse otro estudio imagenológico. Durante la cirugía se identificó el saco herniario no reductible, que en su interior presentaba una estructura tubular correspondiente al apéndice cecal, el cual medía aproximadamente $11 \mathrm{~mm}$ de diámetro, y que mostraba cambios de aspecto flegmonoso en su extremo distal. Se realizó un abordaje pre-peritoneal con ampliación del ligamento lacunar (Ligamento de Gimbernat), posteriormente reducción, apertura y resección del saco herniario. A continuación, se efectuó una apendicetomía con meso (-Fig. 3) y cierre del peritoneo, para finalizar con

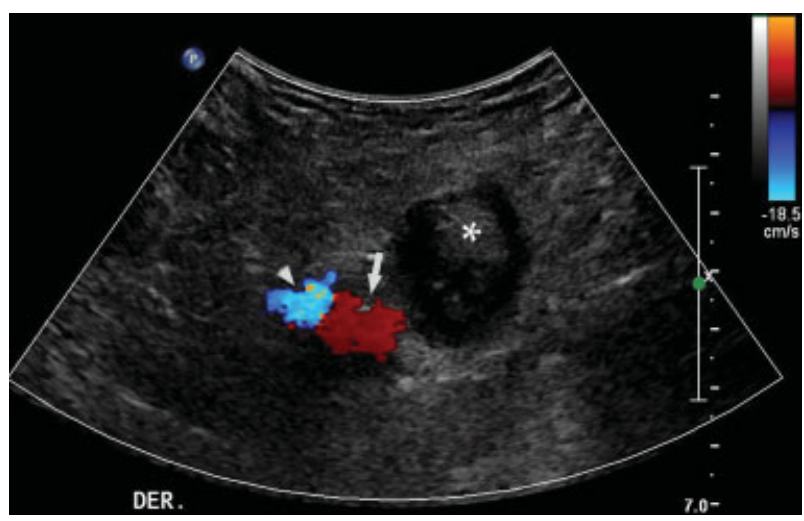

Fig. 1 Ecografía en eje transversal, se visualiza el saco herniario (asterisco) hacia caudal y medial de la vena femoral común (flecha) y arteria femoral común (punta de flecha). Se debe aclarar que en esta imagen existe una inversión de los colores habituales al modo Doppler color (vena en rojo y arteria en azul). Janeiro, Brazil. Todos los derechos reservados.

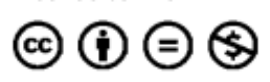

Copyright @ 2019, Sociedad Argentina de Radiología. Publicado por Thieme Revinter Publicações Ltda., Rio de

\section{License terms} $10.1055 / \mathrm{s}-0038-1639487$ ISSN 1852-9992. 


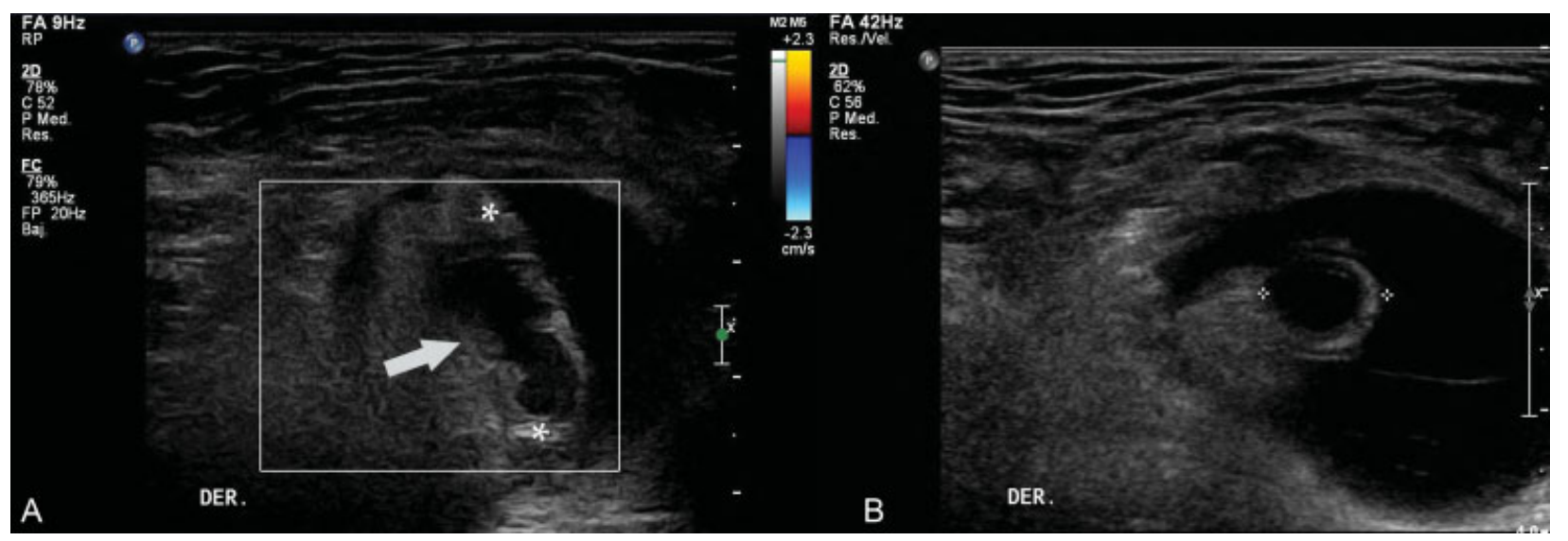

Fig. 2 Ecografía en corte longitudinal del saco herniario (A), el cual contiene una estructura tubular no vascularizada al modo Doppler color (flecha), correspondiente al apéndice cecal, asociado a un aumento de la ecogenicidad del tejido adiposo circundante (asteriscos), compatible con cambios inflamatorios. Corte transversal de la misma estructura tubular (B), la cual alcanza un diámetro máximo de 10 mm.

hernioplastía primaria con dos puntos al anillo crural. La biopsia confirmó que la estructura extraída correspondía al apéndice vermiforme con hiperplasia de la mucosa, periapendicitis con hemorragia reciente $y$ signos de organización. La paciente evolucionó en buenas condiciones, sin complicaciones postoperatorias.

El diagnóstico de la hernia de De Garengeot se ha descrito principalmente como un hallazgo intraoperatorio, existiendo pocos reportes donde el diagnóstico se haya realizado por métodos imagenológicos pre-quirúrgicos, existiendo más reportes en tomografía computada y resonancia magnética ${ }^{2-4}$ que en ultrasonido. ${ }^{5,6}$ Nuestro caso demuestra la gran utilidad de esta última técnica en el diagnóstico, siendo una herramienta ampliamente

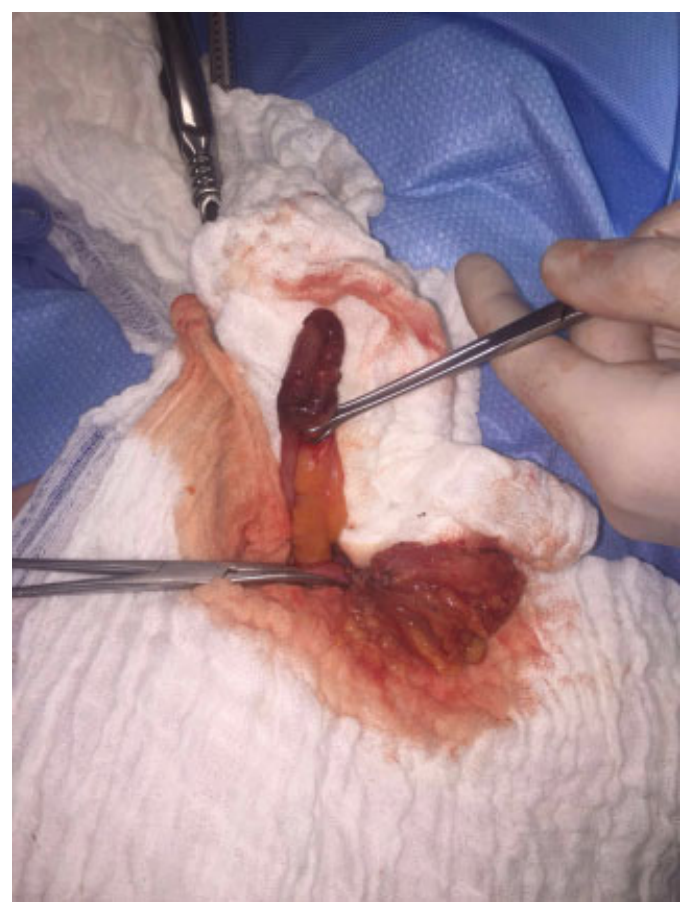

Fig. 3 Pieza quirúrgica. Extracción del apéndice cecal durante procedimiento quirúrgico, el cual se encontraba aumentado de tamaño, con aspecto flegmonoso hacia la punta. disponible y económica. Los criterios imagenológicos en las distintas modalidades de estudio son los mismos que para la apendicitis aguda de la cavidad abdominal; diámetro mayor a $6 \mathrm{~mm}$ en eje transverso, inflamación del tejido adiposo adyacente y/o líquidoperi-apendicular entre otros. En cuanto al tratamiento, sigue siendo un tema de controversia si es un hallazgo en los casos no complicados; sin embargo, cuando se presenta en conjunto con apendicitis aguda la recomendación es manejo quirúrgico, sin existir consenso acerca de si realizar hernioplastia primaria o secundaria ni la técnica a realizar. ${ }^{4,7}$ En nuestro caso se optó por hernioplastia en el mismo momento quirúrgico, sin complicaciones post operatorias tempranas.

\section{Confidencialidad de los datos}

Los autores declaran que han seguido los protocolos de su centro de trabajo sobre la publicación de datos de pacientes y que todos los pacientes incluidos en el estudio han recibido información suficiente y han dado su consentimiento informado por escrito para participar en dicho estudio.

\section{Conflicto de intereses}

Los autores del trabajo declaran no tener ningún conflicto de intereses.

\section{Bibliografía}

1 Croissant de Garengeot R. Traite des operations de chirurgie. París: Cavelier; 1731:369-371

2 Kalles V, Mekras A, Mekras D, et al. De Garengeot's hernia: a comprehensive review. Hernia 2013;17(02):177-182

3 Allen BC, Kirsch J, Szomstein S. Case 187: De Garengeot hernia. Radiology 2012;265(02):640-644

4 Pan CW, Tsao MJ, Su MS. A case of De Garengeot hernia requiring early surgery. BMJ Case Rep 2015;2015:bcr2015211102

5 Filatov J, Ilibitzki A, Davidovitch S, Soudack M. Appendicitis within a femoral hernia: sonographic appearance. J Ultrasound Med 2006;25(09):1233-1235

6 Hao J, Yao J, Guo D, Sun W, Liang J, Jiang X. De Garengeot hernia: the ultrasound and computed tomographic findings in an 81year-old woman. Am J Emerg Med 2014;32(05):486.e5-486.e6

7 Madiha A, Rares H, Abdus S. De Garengeot hernia: a forgotten rare entity? BMJ Case Rep 2014;2014:bcr2013201413 